\title{
Distractor interference stays constant despite variation in working memory load
}

\author{
Zhe Chen and Celestien C. Chan \\ University of Canterbury, Christchurch, New Zealand
}

\begin{abstract}
Previous studies have shown that working memory (WM) plays an important role in selective attention, so that high WM load leads to inefficient distractor inhibition, in comparison with low WM load. In the present study, we examined the effect of WM on distractor processing while the extent of attentional focus was held constant. Our results show that WM load affected distractor processing only when it was positively correlated with the extent of attentional focus. When the latter was held constant, the effect of WM became negligible. Furthermore, when low WM load was paired with a wide attentional focus and high WM load was matched with a narrow attentional focus, greater distractor processing was found when the WM load was low than when it was high. These results suggest that efficient distractor inhibition may require only minimal WM resources and that the effect of WM on distractor processing is more complex than has previously been assumed.
\end{abstract}

An important task for vision scientists is to understand how the visual system selects relevant information among competing distractors. Prior research has identified several factors that influence the efficiency of selective attention. These factors include the spatial proximity and perceptual grouping between a target and distractors (B. A. Eriksen \& C. W. Eriksen, 1974; Kramer \& Jacobson, 1991), the extent of attentional focus induced by a task (Chen, 2000, 2003; C. W. Eriksen \& St. James, 1986; LaBerge, Brown, Carter, Bash, \& Hartley, 1991), and the perceptual load involved in the processing of a target (Lavie, 1995).

In addition to the factors above, recent research suggests that the extent of distractor processing is also influenced by working memory (WM) capacity. Several studies have reported a positive correlation between WM span and distractor inhibition. Relative to participants with low WM span, participants with high WM span showed less Stroop interference (Kane \& Engle, 2003), were faster to perform antisaccade tasks, and made fewer reflexive saccades to an exogenous cue on the wrong side of the screen (Unsworth, Schrock, \& Engle, 2004).

There is evidence that WM may even play a causal role in distractor inhibition. Lavie and her colleagues (Lavie, Hirst, de Fockert, \& Viding, 2004) conducted several experiments in which participants performed a letter discrimination task while holding either one digit (low WM load) or several digits (high WM load) in memory. Distractor interference was larger in the high-load condition than in the low-load condition. Similar results were obtained when the task was to search for a target among irrelevant items in the presence of a unique singleton distractor (Lavie \& de Fockert, 2005). The distractor caused more interference when the search was performed with a concurrent memory task than when it was performed without one.

The effect of WM on distractor processing is consistent with the load theory of attention proposed by Lavie (see Lavie, 2005, for a review). According to the theory, selective attention involves two mechanisms: a perceptual selection mechanism that prevents distractor processing under situations of high perceptual load and a cognitive control mechanism that inhibits distractor interference under situations of low perceptual load. Specifically, when distractors have been perceived due to low perceptual load, the efficiency of distractor inhibition depends on the availability of executive cognitive control functions. When WM, which is part of the executive cognitive control mechanism, is loaded, efficient distractor inhibition will be impaired, due to the lack of resources available to actively maintain stimulus-processing priorities. As a result, distractor interference will be greater when WM load is high than when it is low.

However, despite empirical support for the cognitive control account of distractor inhibition from several studies (Lavie \& de Fockert, 2005; Lavie et al., 2004), other experiments have shown conflicting results (Logan, 1978; Woodman, Vogel, \& Luck, 2001). Logan reported no difference in visual search slopes when his participants searched for targets either with or without a concurrent memory task. Woodman et al. also observed comparable search slopes in trials with different visual WM loads. These results suggest that high WM load does not always lead to impaired performance in selective attention.

These seemingly inconsistent results raise an interesting question regarding the relationship between WM and distractor inhibition. Is it possible that the effect of WM 
load on distractor inhibition is modulated by some other factors? The goal of the present study was to examine the role of WM in selective attention while controlling the extent of attentional focus. In prior studies, a common way to manipulate WM load was to vary the number of items the participants held in memory while they performed a selective attention task. When the to-be-remembered items were presented simultaneously, the number of items would be correlated positively with the extent of attentional focus. Consequently, high WM load was typically associated with a wide attentional focus, whereas low load was associated with a narrow attentional focus (e.g., Lavie \& de Fockert, 2005; Lavie et al., 2004). Because attentional focus is known to influence the efficiency of distractor inhibition (Chen, 2000, 2003; C. W. Eriksen \& St. James, 1986; LaBerge et al., 1991), the systematic pairing between WM load and attentional focus raises the possibility that the differential distractor interference in the low versus high WM load conditions may be explained by a difference in attentional focus.

In the experiments reported here, we assessed the role of WM load in several ways. In Experiment 1A, we covaried WM load with the extent of attentional focus so that they were positively correlated. In Experiments 1B and $1 \mathrm{C}$, we manipulated WM load while holding constant the extent of attentional focus and vice versa. In Experiment 2, we pitted one factor against the other. Finally, in Experiment 3, we increased WM load, to ensure that our results could be generalized to other levels of WM load. We found no evidence that WM load affected the degree of distractor inhibition. Instead, distractor interference differed as a function of the extent of attentional focus.

\section{EXPERIMENTS 1A, 1B, AND 1C}

In Experiment 1A, we deliberately covaried WM load with the extent of attentional focus, so that a high WM load was paired with a wide attentional focus and a low
WM load with a narrow attentional focus. Our goal was to establish differential levels of distractor interference across the critical experimental conditions. We expected greater distractor interference in the high-load/wide-focus condition than in the low-load/narrow-focus condition. Such a result would be predicted by both the cognitive control account and the attentional focus account of distractor inhibition.

In Experiment 1B, we tested the cognitive control account of distractor inhibition by varying WM load while controlling the extent of attentional focus. The cognitive control account would predict larger distractor interference on high- than on low-load trials. In Experiment 1C, we examined the attentional focus account by manipulating the extent of attentional focus while keeping WM load constant. If attentional focus is a major determinant of distractor interference, greater distractor interference should be found in the wide-focus condition than in the narrow-focus condition.

\section{Experiment 1A}

\section{Method}

Participants. Fourteen undergraduate students from the University of Canterbury took part in the experiment in exchange for payment. ${ }^{1}$

Stimuli and Design. All the stimuli were presented against a homogenous gray background. Each trial consisted of a fixation, a cue, and a target display (see Figure 1). The fixation was a small white cross at the center of the screen. The cue was made of either one square that subtended $0.57^{\circ}$ or four identical squares that formed a $7.54^{\circ} \times 7.93^{\circ}$ imaginary rectangle. The cue was equally likely to be red, green, or white and on the left or right side of the screen, with its center $4.59^{\circ}$ from the fixation. The target display consisted of a black target letter (H or S; size: 48) surrounded by four identical black distractor letters ( $\mathrm{H}, \mathrm{S}$, or X; size: 72). The entire display subtended $7.07^{\circ}$.

The experiment was a $2 \times 2$ within-subjects design, with the principal manipulations being target-distractor compatibility (neutral vs. incongruent) and WM load/attentional focus (high-load/wide-focus vs. low-load/narrow-focus). Whereas target-distractor compatibility
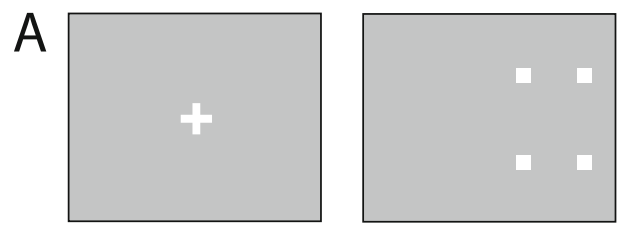

B

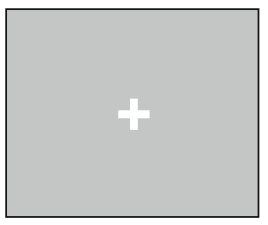

Fixation

$(1,005 \mathrm{msec})$

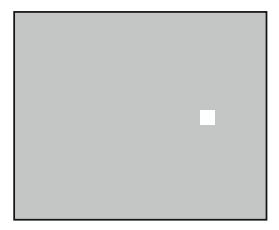

Cue

(120 msec)

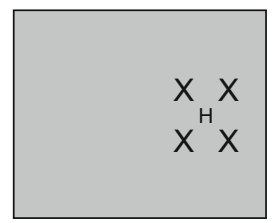

Respond to
Target Letter

Respond to

Cue Color

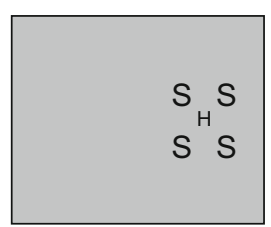

Respond to

Target Letter

Press the

Designated Key

Target

(120 msec)

\section{Selective Memory Task Attention Task (Until Response) (Until Response)}

Figure 1. Examples of (A) a typical trial in the high-load/wide-focus condition and (B) a typical trial in the lowload/narrow-focus condition in Experiment 1A. 
was varied within blocks, load/focus was varied across blocks, with block order counterbalanced across participants. On half the trials, the distractors were not associated with the target response, and on the rest of the trials, they indicated a response different from that for the target. In the high-load/wide-focus condition, the cue was made of four squares, and the task was speeded letter discrimination, followed by an accuracy-only response for the color of the cue. In the low-load/narrow-focus condition, the cue was made of one square, and in addition to the letter discrimination task, the participants pressed a designated key to initiate the next trial, irrespective of the color of the cue.

The fixation was presented for $1,005 \mathrm{msec}$, followed by a $120-\mathrm{msec}$ display of the cue on the left or right side of the screen with equal probability. The target display then appeared for $120 \mathrm{msec}$ on the same side of the screen as the cue. For the letter discrimination task, the two designated keys were "> " for $\mathrm{H}$ and "/" for S. For the memory task, the participants pressed one of three labeled keys (" $z$ " for white, " $\mathrm{x}$ " for red, and "c" for green), in accordance with the color of the cue, in the high-load condition or the " $\mathrm{z}$ " key, regardless of the color of the cue, in the low-load condition.

\section{Results}

Accuracy for the memory task was high, with the mean memoryrelated error rates being $4.5 \%$ and $0 \%$ for the high-load/wide-focus and low-load/narrow-focus conditions, respectively. ${ }^{2}$ The results for the letter discrimination task are shown in Table 1, Experiment 1A. A repeated measures ANOVA on mean reaction time (RT) and accuracy data showed faster and more accurate responses on the neutral trials (591 msec with $1.6 \%$ error) than on the incongruent ones $\left(619 \mathrm{msec}\right.$ with $4.0 \%$ error) $\left[F(1,13)=52.21, M S_{\mathrm{e}}=207, p<\right.$ .001 , and $F(1,13)=11.37, M S_{\mathrm{e}}=6.76, p<.01$, for RT and accuracy, respectively] and faster responses in the low-load/narrow-focus condition $(490 \mathrm{msec})$ than in the high-load/wide-focus condition $(720 \mathrm{msec})\left[F(1,13)=45.78, M S_{\mathrm{e}}=16,159, p<.001\right]$. Furthermore, the compatibility effect was larger in the high-load/wide-focus condition $(34 \mathrm{msec})$ than in the low-load/narrow-focus condition $(22 \mathrm{msec})\left[F(1,13)=5.11, M S_{\mathrm{e}}=101, p<.05\right]$. No other effects reached significance. (See Experiment $1 \mathrm{C}$ for the discussion of the results of this experiment and those of Experiment 1B.)

\section{Experiment 1B}

\section{Method}

In Experiment 1B $(N=10)$, WM load was manipulated while the extent of attentional focus was held constant. The method was identical to that in Experiment 1A, except that the cue was always made of four squares. As before, after letter discrimination, the participants reported the color of the cue in the high-load condition but pressed a designated key, regardless of the color of the cue, in the low-load condition.

\section{Results}

Accuracy for the memory task was again high (see Table 1, Experiment 1B). The mean memory-related error rates for the high- and low-load conditions were $6 \%$ and $0 \%$, respectively. For the letter discrimination task, the participants were again faster and more accurate on the neutral trials (624 msec with $2.8 \%$ error) than on the incongruent ones (657 msec with $5.7 \%$ error) $[F(1,9)=29.89$, $M S_{\mathrm{e}}=352.7, p<.001$, and $F(1,9)=21.97, M S_{\mathrm{e}}=3.99$, $p<.01$, for RT and accuracy, respectively]. They were also faster when the load was low $(502 \mathrm{msec})$ than when it was high $(779 \mathrm{msec})\left[F(1,9)=14.46, M S_{\mathrm{e}}=53,060.4\right.$, $p<.01]$. However, the critical compatibility $\times$ load interaction was not significant for either RT $[F(1,9)=0.01$, $M S_{\mathrm{e}}=278.7$, n.s. $]$ or accuracy $\left[F(1,9)=1.75, M S_{\mathrm{e}}=\right.$ 3.39 , n.s.], suggesting a negligible WM load effect. No other effects were found.

\section{Experiment 1C}

\section{Method}

In Experiment 1C $(N=14)$, the extent of attentional focus was varied while WM load was controlled for. Unlike in the previous experiments, the participants performed a single task of letter discrimination. Because cue validity was $100 \%$ with respect to target location and abrupt onsets capture attention (Yantis \& Jonides,

Table 1

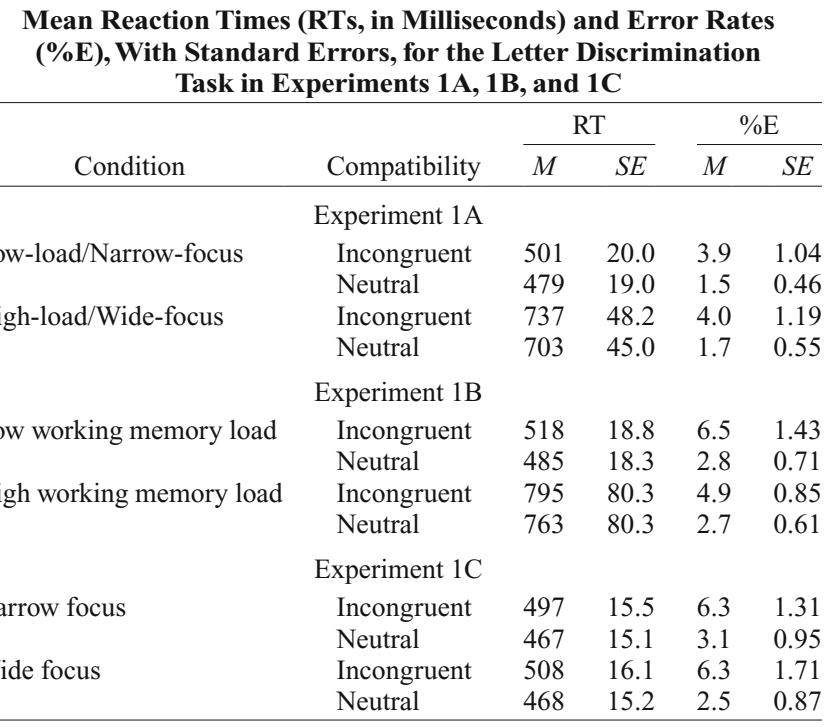

Note-The standard errors shown here represent the between-subjects variability within a condition, not the within-subjects variability across conditions that is of interest here. 
1984), we assumed that the participants would attend to the cue and that the two types of cues would induce different sizes of attentional focus. The cue was always white and consisted of four squares in the wide-focus condition and a single square in the narrow-focus condition. All other aspects of the method were identical to those in Experiment 1A.

\section{Results and Discussion}

The compatibility effect was again significant, with faster and more accurate responses on the neutral trials (468 $\mathrm{msec}$ with 2.8\% error; see Table 1, Experiment 1C) than on the incongruent ones $(503 \mathrm{msec}$ with $6.3 \%$ error $)[F(1,13)=$ $103.4, M S_{\mathrm{e}}=165, p<.001$ for RT, and $F(1,13)=16.14$, $M S_{\mathrm{e}}=10.7, p<.01$, for accuracy]. More important, there was a size $\times$ compatibility interaction for $\mathrm{RT}[F(1,13)=$ $\left.6.05, M S_{\mathrm{e}}=58, p<.05\right]$. The compatibility effect was larger when the cue was wide $(40 \mathrm{msec})$ than when it was narrow $(30 \mathrm{msec})$. No other effects were significant.

Consistent with prior research (Lavie \& de Fockert, 2005; Lavie et al., 2004), Experiment 1A showed greater distractor interference when WM load was high than when it was low. However, as we stated earlier, because the level of WM load was confounded with the extent of attentional focus, this result could have been caused by a difference in WM load and/or a difference in the extent of attentional focus.

Interestingly, when the extent of attentional focus was controlled in Experiment 1B, the previously observed differential compatibility effect disappeared. Although WM load increased the overall RT for the letter discrimination task, it did not increase the amount of distractor interference. In other words, the magnitude of the distractor compatibility effect remained constant in both the low and the high WM load conditions, suggesting that variation in WM does not influence the degree of distractor interference. What is more, the differential compatibility effect was reinstated in Experiment $1 \mathrm{C}$ when attentional focus was varied while WM load was held constant. Taken together, these findings were consistent with the attentional focus account, rather than with the cognitive control account, of distractor inhibition.

\section{EXPERIMENT 2}

Experiment $2(N=14)$ was a critical experiment. It was designed to pit the cognitive control account against the attentional focus account. The method was the same as that in Experiment 1A, except for one crucial difference: High WM load was paired with a narrow attentional focus, whereas low WM load was paired with a wide attentional focus. Whereas the cognitive control account would predict a larger compatibility effect in the high-load/narrowfocus condition than in the low-load/wide-focus condition, the attentional focus account would predict the opposite: greater distractor interference in the low-load/wide-focus condition than in the high-load/narrow-focus condition.

\section{Results and Discussion}

As in the previous experiments, the participants showed high accuracy for the memory task, with the memoryrelated error rates being $6.9 \%$ and $0 \%$ for the high-load/ narrow-focus and low-load/wide-focus conditions, respectively. Table 2 shows the data for the letter discrimination task. An ANOVA on RT revealed significant effects of load/focus $\left[F(1,13)=16.11, M S_{\mathrm{e}}=24,336, p<.01\right]$ and compatibility $\left[F(1,13)=24.8, M S_{\mathrm{e}}=389, p<.01\right]$ and a significant interaction $\left[F(1,13)=9.84, M S_{\mathrm{e}}=151\right.$, $p<.01]$, with a larger compatibility effect in the lowload/wide-focus condition $(37 \mathrm{msec})$ than in the highload/narrow-focus condition (16 msec). A similar analysis on accuracy showed lower error rates on the neutral trials than on the incongruent trials $\left[F(1,13)=5.23, M S_{\mathrm{e}}=\right.$ $9.51, p<.04]$. No other effects were significant.

These results were inconsistent with the cognitive control interpretation of distractor inhibition, which would predict greater distractor processing under high WM load than under low WM load. Once again, the results suggest that attentional focus, rather than WM load, played a critical role in selective attention, at least in the present paradigm.

Admittedly, there was one important methodological difference between the present experiments and those in prior research. Whereas high WM load involved maintaining one item in memory in our experiments, it typically has involved holding multiple items in memory in other studies (e.g., Lavie et al., 2004). Because WM capacity is generally believed to be four items (Cowan, 2001), it is possible that our results were caused by an underuse of WM capacity. Experiment 3 addressed this possibility by increasing overall WM load.

Table 2

Mean Reaction Times (RTs, in Milliseconds) and Error Rates $(\% \mathrm{E})$, With Standard Errors, for the Letter Discrimination Task in Experiment 2

\begin{tabular}{|c|c|c|c|c|c|}
\hline \multirow[b]{2}{*}{ Condition } & \multirow[b]{2}{*}{ Compatibility } & \multicolumn{2}{|c|}{ RT } & \multicolumn{2}{|c|}{$\% \mathrm{E}$} \\
\hline & & $M$ & $S E$ & $M$ & $S E$ \\
\hline \multirow[t]{2}{*}{ Low-load/Wide-focus } & Incongruent & 518 & 13.0 & 5.2 & 1.07 \\
\hline & Neutral & 481 & 12.9 & 2.1 & 0.47 \\
\hline \multirow[t]{2}{*}{ High-load/Narrow-focus } & Incongruent & 675 & 42.3 & 3.3 & 0.77 \\
\hline & Neutral & 659 & 43.9 & 2.7 & 0.57 \\
\hline
\end{tabular}

Note-The standard errors shown here represent the between-subjects variability within a condition, not the within-subjects variability across conditions that is of interest here. 

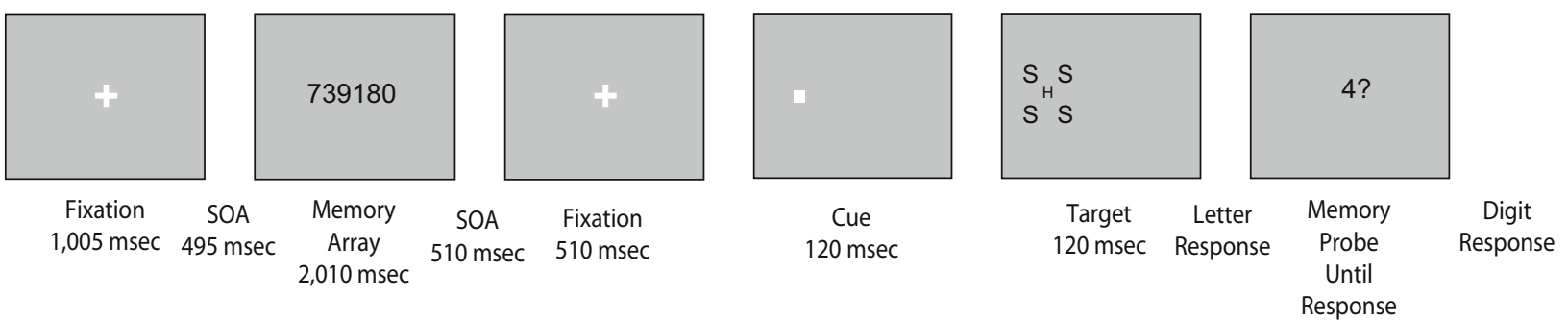

Figure 2. An example of a typical trial in the high-load/narrow-focus condition in Experiment 3. In both the low-load/narrowfocus and the low-load/wide-focus conditions, the presentation duration of the memory array was 510 msec. SOA, stimulus onset asynchrony.

\section{EXPERIMENT 3}

\section{Method}

Fifty-four new participants were recruited. They were equally divided and randomly assigned to one of three groups: high-load/ narrow-focus, low-load/narrow-focus, and low-load/wide-focus. All performed a letter discrimination task, followed by a digit memory task.

Several changes were made in methodology (see Figure 2). First, a memory array was inserted after the fixation. It consisted of either one digit or six different digits. Second, the cue, which could be small (one square) or large (four squares), was always white. Third, a memory probe that consisted of one digit and a question mark was presented immediately after the participants performed the letter discrimination task. On half the trials, the memory probe was in the memory array, and on the remaining trials, it was absent from it. The participants pressed the " $\mathrm{z}$ " or " $\mathrm{x}$ " key for the probe-present and probe-absent trials, respectively.

\section{Results and Discussion}

Tables 3A and 3B show the data. Two participants' data were excluded from analyses due to their high error rates, which exceeded $30 \%$ in one condition. Two mixed ANOVAs with target-distractor compatibility as the within-subjects factor and group as the between-subjects factor were conducted on the RT and accuracy data. The participants were faster and more accurate on the neutral than on the incongruent trials $\left[F(1,49)=103.39, M S_{\mathrm{e}}=\right.$ $122, p<.0001$, and $F(1,49)=10.91, M S_{\mathrm{e}}=4.83, p<$ .001 , for RT and accuracy, respectively]. In addition, for RT, there was a significant effect of group $[F(2,49)=$ 3.27, $\left.M S_{\mathrm{e}}=16,411, p<.05\right]$ and a compatibility $\times$ group interaction $\left[F(2,49)=5.17, M S_{\mathrm{e}}=122, p<.01\right]$. No other effects reached significance.

To clarify the interaction for RT, we conducted two additional ANOVAs. A comparison between the high-load/ narrow-focus group and the low-load/narrow-focus group allowed us to assess the effect of WM load. The analysis revealed a significant compatibility effect $[F(1,33)=$ $\left.68.75, M S_{\mathrm{e}}=75, p<.0001\right]$ but no compatibility $\times$ group interaction $\left[F(1,33)=0.09, M S_{\mathrm{e}}=75\right.$, n.s.], suggesting that WM load had little influence on distractor inhibition. Likewise, a comparison between the low-load/narrowfocus group and the low-load/wide-focus group allowed us to measure the effect of attentional focus. Importantly, in addition to a significant compatibility effect $[F(1,33)=$ $\left.74.97, M S_{\mathrm{e}}=137, p<.0001\right]$, a reliable group $\times$ compatibility interaction was found $\left[F(1,33)=7.62, M S_{\mathrm{e}}=\right.$ $137, p<.01]$, indicating that attentional focus affected distractor inhibition. No other effects were reliable.

An ANOVA on memory error rates showed a significant group difference $\left[F(2,49)=4.55, M S_{\mathrm{e}}=23.22, p<\right.$ $.05]$. Tukey's HSD tests further indicated higher error rates for the high-load/narrow-focus group (10.04\%) than for both the low-load/narrow-focus and the low-load/widefocus groups $(5.76 \%$ and $5.72 \%$, respectively), with no significant difference between the latter two. These results suggest that our manipulation of WM load was effective.

The most important finding of the experiment was the observation of the differential compatibility effect as a function of attentional focus, instead of WM load. This result was important because it showed that the findings

Table 3A

Mean Reaction Times (RTs, in Milliseconds) and Error Rates $(\% \mathrm{E})$, With Standard Errors, for the Letter Discrimination Task in Experiment 3

\begin{tabular}{|c|c|c|c|c|c|}
\hline \multirow[b]{2}{*}{ Condition } & \multirow[b]{2}{*}{ Compatibility } & \multicolumn{2}{|c|}{ RT } & \multicolumn{2}{|c|}{$\% \mathrm{E}$} \\
\hline & & $M$ & $\overline{S E}$ & $M$ & $S E$ \\
\hline \multirow[t]{2}{*}{ High-load/Narrow-focus } & Incongruent & 554 & 22.5 & 4.8 & 1.03 \\
\hline & Neutral & 536 & 21.0 & 3.3 & 0.85 \\
\hline \multirow[t]{2}{*}{ Low-load/Narrow-focus } & Incongruent & 577 & 19.1 & 5.4 & 0.97 \\
\hline & Neutral & 560 & 19.3 & 3.2 & 0.70 \\
\hline \multirow[t]{2}{*}{ Low-load/Wide-focus } & Incongruent & 639 & 24.2 & 4.6 & 0.93 \\
\hline & Neutral & 607 & 24.8 & 4.0 & 0.81 \\
\hline
\end{tabular}

Note-The standard errors shown here represent the between-subjects variability within a condition, not the within-subjects variability across conditions that is of interest here. 
Table 3B

Error Rates (Percent Incorrect)

for the Memory Task in Experiment 3

\begin{tabular}{cccc}
\hline & $\begin{array}{c}\text { High-Load/ } \\
\text { Narrow-Focus }\end{array}$ & $\begin{array}{c}\text { Low-Load/ } \\
\text { Narrow-Focus }\end{array}$ & $\begin{array}{c}\text { Low-Load/ } \\
\text { Wide-Focus }\end{array}$ \\
\hline \% Error & 10.04 & 5.76 & 5.72 \\
\hline
\end{tabular}

of our previous experiments were not limited to situations of low WM load. It also eliminated a potentially important methodological difference between the present research and prior studies (e.g., Lavie et al., 2004). Taken together, our data support the attentional focus account, rather than the WM load account, of distractor inhibition.

\section{GENERAL DISCUSSION}

In the present study, the efficiency of distractor inhibition was not influenced by WM load when the extent of attentional focus was controlled. Of the four experiments in which WM load was manipulated, only Experiment 1A showed a greater compatibility effect in the high- than in the low-load condition. However, because WM load varied systematically with attentional focus, the differential compatibility effect was equally likely to be caused by a difference in attentional focus as by a difference in WM load. Importantly, when attentional focus was controlled, the effect of WM load on distractor interference disappeared. In contrast, when WM load was held constant, attentional focus affected selective attention, so that the compatibility effect was larger in the wide-focus condition than in the narrow-focus one. These results were observed regardless of whether the overall WM load was relatively low (one item vs. zero items) or high (six items vs. one item). Furthermore, when low WM load was paired with a wide attentional focus and high WM load with a narrow attentional focus, the participants showed a larger compatibility effect for the former than for the latter. These findings are difficult to reconcile with the cognitive control account of distractor inhibition.

Our results are also consistent with the findings of Logan (1978). As we noted earlier, Logan did not find an effect of WM load on the visual search slopes. Interestingly, although the issue of attentional focus was not examined explicitly in the study, the extent of attentional focus was held constant across the different load conditions, because the stimuli in the memory array were presented sequentially. This ensured that there was no systematic variation in the extent of attentional focus across the different load conditions.

One may wonder why our results are inconsistent with the findings of de Fockert, Rees, Frith, and Lavie (2001), who presented an equal number of elements in the memory array across the different load conditions but still found greater interference on the high- than on the lowload trials. In de Fockert et al., the task was to categorize famous names (pop stars vs. politicians) that were superimposed on congruent, incongruent, or neutral distractor faces while concurrently holding in memory four digits. Whereas the digits were always in a fixed order (i.e.,
1234 ) in the low WM load condition, they were in a random order on each trial (e.g., 9476 ) in the high WM load condition. A larger compatibility effect was found when the WM load was high than when it was low.

Although the results of de Fockert et al. (2001) are consistent with the cognitive control account of distractor inhibition, there may exist an alternative interpretation. Unlike in our experiments, in which we used $100 \%$ valid cues to indicate the spatial location of the target as a means of inducing the participants to pay attention to the cue, thereby controlling their attentional focus, de Fockert et al. did not employ any explicit means to control the participants' attentional focus. Because the trials in the low and high memory load conditions were blocked, it would have been unlikely for the participants in de Fockert et al.'s study to adopt similar extents of attentional focus in the two conditions, since there was no need to pay attention to the digits in the low-load condition, they were the same throughout the entire block. The lack of control in the extent of attentional focus could then lead to differential distractor interference in the low- and the high-load conditions.

To conclude, the present research revealed no evidence that the degree of distractor interference varied as a function of WM load when the extent of attentional focus was controlled. However, this does not necessarily mean that selective attention does not require WM at all. It is entirely possible that a complete depletion of WM may result in impaired performance, just as a complete depletion of attentional resources can impair feature search even though feature search does not appear to require attention under most circumstances (Joseph, Chun, \& Nakayama, 1997). It is also possible that the effect of WM load on distractor processing depends on the specific types of memory and selective attention tasks. Indeed, recent experiments by Woodman and Luck (2004) show that maintaining spatial information in WM impairs visual search efficiency, even though maintaining object representations in WM does not (Woodman et al., 2001). Whether the effect of WM on selective attention is task specific and how many WM resources are necessary for efficient distractor inhibition require further investigation.

\section{AUTHOR NOTE}

This research was supported by Grant U6498 from the University of Canterbury to Z.C. We thank Anne Hillstrom and the reviewers for helpful comments on an early version of the manuscript. Correspondence concerning this article should be addressed to Z. Chen, Department of Psychology, University of Canterbury, Private Bag 4800, Christchurch, New Zealand (e-mail: zhe.chen@canterbury.ac.nz).

Note-Accepted by David A. Balota's editorial team.

\section{REFERENCES}

Chen, Z. (2000). An object-based cost of visual filtering. Perception \& Psychophysics, 62, 482-495.

Chen, Z. (2003). Attention focus, processing load, and Stroop interference. Perception \& Psychophysics, 65, 888-900.

Cowan, N. (2001). The magical number 4 in short-term memory: A reconsideration of mental storage capacity. Behavioral \& Brain Sciences, 24, 87-114.

De Fockert, J. W., Rees, G., Frith, C. D., \& Lavie, N. (2001). The role of working memory in visual selective attention. Science, 291, 1803-1806. 
EriKsen, B. A., \& ERIKSEN, C. W. (1974). Effects of noise letters upon the identification of a target letter in a nonsearch task. Perception \& Psychophysics, 16, 143-149.

Eriksen, C. W., \& St. James, J. D. (1986). Visual attention within and around the field of focal attention: A zoom lens model. Perception \& Psychophysics, 40, 225-240.

JosePh, J. S., Chun, M. M., \& NAKAYAma, K. (1997). Attentional requirements in a 'preattentive' feature search task. Nature, 387, 805-807.

Kane, M. J., \& EngLe, R. W. (2003). Working-memory capacity and the control of attention: The contributions of goal neglect, response competition, and task set to Stroop interference. Journal of Experimental Psychology: General, 132, 47-70.

Kramer, A. F., \& Jacobson, A. (1991). Perceptual organization and focused attention: The role of objects and proximity in visual processing. Perception \& Psychophysics, 50, 267-284.

LaBerge, D., Brown, V., Carter, M., Bash, D., \& Hartley, A. (1991). Reducing the effects of adjacent distractors by narrowing attention. Journal of Experimental Psychology: Human Perception \& Performance, 17, 65-76.

LaVIE, N. (1995). Perceptual load as a necessary condition for selective attention. Journal of Experimental Psychology: Human Perception \& Performance, 21, 451-468.

LAviE, N. (2005). Distracted and confused? Selective attention under load. Trends in Cognitive Sciences, 9, 75-82.

LAVIE, N., \& DE FocKerT, J. [W.] (2005). The role of working memory in attentional capture. Psychonomic Bulletin \& Review, 12, 669-674.

Lavie, N., Hirst, A., De Fockert, J. W., \& Viding, E. (2004). Load theory of selective attention and cognitive control. Journal of Experimental Psychology: General, 133, 339-354.

LogAN, G. D. (1978). Attention in character-classification tasks: Ev- idence for the automaticity of component stages. Journal of Experimental Psychology: General, 107, 32-63.

Unsworth, N., Schrock, J. C., \& Engle, R. W. (2004). Working memory capacity and the antisaccade task: Individual differences in voluntary saccade control. Journal of Experimental Psychology: Learning, Memory, \& Cognition, 30, 1302-1321.

Woodman, G. F., \& LucK, S. J. (2004). Visual search is slowed when visuospatial working memory is occupied. Psychonomic Bulletin \& Review, 11, 269-274.

Woodman, G. F., Vogel, E. K., \& Luck, S. J. (2001). Visual search remains efficient when visual working memory is full. Psychological Science, 12, 219-224.

YANTIS, S., \& Jonides, J. (1984). Abrupt visual onsets and selective attention: Evidence from visual search. Journal of Experimental Psychology: Human Perception \& Performance, 10, 601-621.

\section{NOTES}

1. The participants' mean ages for the different experiments were 20.4, 20.8, 22.6, 23.4, and 20.7 years for Experiments 1A, 1B, 1C, 2, and 3, respectively. No participant took part in more than one experiment.

2. On a small proportion of trials in the memory task, instead of pressing one of the response keys designated for the memory task, some participants would press a key designated for the letter discrimination task or a key unrelated to any task. We consider this type of error as a response error, rather than a memory error. The mean error rates for response errors were $1.8 \%, 0.8 \%$, and $2.8 \%$ for Experiments $1 \mathrm{~A}, 1 \mathrm{~B}$, and 2, respectively.

(Manuscript received October 18, 2005; revision accepted for publication May 23, 2006.) 